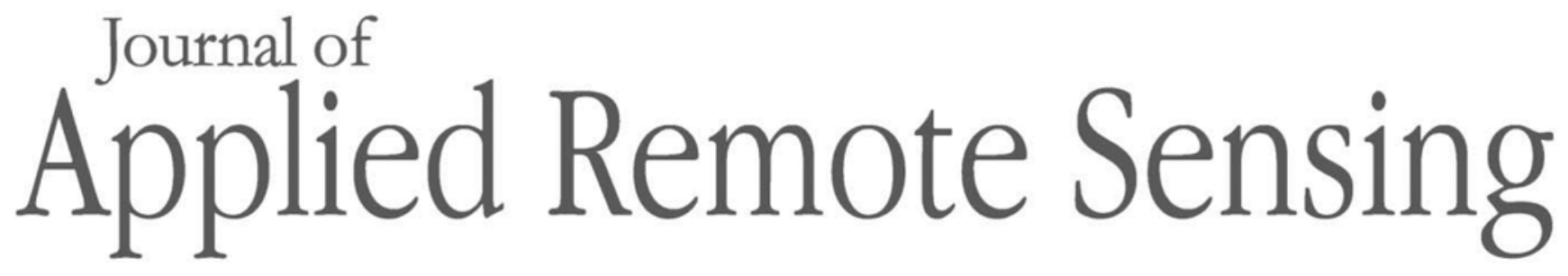

RemoteSensing.SPIEDigitalLibrary.org

\title{
Estimation of biomass and carbon stock in Para rubber plantations using object-based classification from Thaichote satellite data in Eastern Thailand
}

Kitsanai Charoenjit

Pierpaolo Zuddas

Pascal Allemand

Sura Pattanakiat

Katavut Pachana

\section{SPIE.}




\title{
Estimation of biomass and carbon stock in Para rubber plantations using object-based classification from Thaichote satellite data in Eastern Thailand
}

\author{
Kitsanai Charoenjit, ${ }^{a} *$ Pierpaolo Zuddas, ${ }^{a}$ Pascal Allemand, ${ }^{b}$ \\ Sura Pattanakiat, ${ }^{c}$ and Katavut Pachana ${ }^{d}$ \\ ${ }^{a}$ UPMC-Sorbonne Universités, Institut des Sciences de la Terre de Paris, 4 Place Jussieu, \\ 75252 Paris, France \\ bUniversité Claude Bernard Lyon1, Laboratoire de Géologie de Lyon, Terre, Planètes, \\ Environnement (LGL-TPE), 2 rue Raphaël Dubois, 69622 Villeurbanne Lyon, France \\ ${ }^{c}$ Mahidol University, Geo-Informatics in Resource and Environment Research and Training \\ Center (GIREN), Faculty of Environment and Resource Studies, 999 Salaya, Phutthamonthon, \\ Nakhon Pathom 73170, Thailand \\ ${ }^{\mathrm{d} B u r a p h a}$ University, Faculty of Science, Department of Chemistry, 169 Long-Hard Bangsaen \\ Road, Saen Sook Subdistrict, Chonburi 20131, Thailand
}

\begin{abstract}
This paper deals with the efficiency of measurements of carbon stock by remote sensing techniques on Para rubber plantations in Thailand. These plantations could play an important role in carbon budget and thus are part of the Clean Development Mechanism of the Kyoto Protocol. Current methods of carbon stock estimations use middle resolution images and produce results with a large uncertainty. We use very high resolution images from the Thaichote satellite, associated with field measurements to estimate the carbon stock and its evolution in the Mae num Prasae watershed, Eastern Thailand. Using object-based classifications, the plantations have been mapped and their age has been estimated from a parametric model derived from both spectral and textural information and field data. The total biomass and carbon stocked are 2.23 and 0.99 Megaton with an uncertainty of $11 \%$. One hundred and twenty one tons of carbon are sequestered annually in the Para rubber plantations of the studied area. (C) The Authors. Published by SPIE under a Creative Commons Attribution 3.0 Unported License. Distribution or reproduction of this work in whole or in part requires full attribution of the original publication, including its DOI. [DOI: 10 .1117/1.JRS.9.096072]
\end{abstract}

Keywords: carbon stock estimation; object-based classification; Para rubber plantation; Thaichote satellite; Eastern Thailand.

Paper 14701 received Nov. 19, 2014; accepted for publication Feb. 24, 2015; published online Mar. 19, 2015.

\section{Introduction}

$\mathrm{CO}_{2}$ is the most abundant atmospheric gas related to global warming. $\mathrm{CO}_{2}$ is responsible for more than half of the radiative forces associated with the greenhouse effect. ${ }^{1}$ Forest may play an important role in the short carbon dioxide cycle. In particular, tropical forests have the potential capacity to sequester and to conserve carbon permanently., This is why the Clean Development Mechanism recommended by the Kyoto Protocol advocates evaluating tree capacity of $\mathrm{CO}_{2}$ storage in humid tropical forest plantations. ${ }^{4}$ Para rubber is a perennial tree of economic importance in Indonesia, Malaysia, and particularly in Thailand for producing latex for the world-market. The Para rubber has a high biomass, high growth rate, and strong potential for carbon storage. ${ }^{5}$ Thailand is the leader of rubber production in the world; it produces around $37 \%$ of the world's annual rubber production. ${ }^{6}$

Today, very high resolution (VHR) sensors on board satellites can map tropical forest plantations and provide valuable data to evaluate forest biomass and carbon stock evolution. VHR

*Address all correspondence to: Kitsanai Charoenjit, E-mail: kitsanai.charoenjit@upmc.fr 
data has overcome the limitation of spatial resolution of the medium-low resolution sensors such as Landsat 8 OLI $(15 \mathrm{~m})$ or MODIS $(250 \mathrm{~m})$. These sensors cannot capture tree characteristics such as the crown canopy. Therefore, estimations of plantation biomass and carbon stocks from low resolution remote sensing data were inaccurate. ${ }^{7}$ The Thaichote satellite camera (previously named THEOS) is a high resolution sensor with a 2-m resolution. It is the first Earth observation satellite of Thailand. Its data are potentially an important data source for biomass and carbon stock estimation of large surfaces. However, the evaluation of forest carbon stock is a complex task. Numerous approaches have been proposed to estimate biomass using remote sensing techniques. $^{7-13}$ The spectral information contained in satellite data is classically used. ${ }^{10-13}$ The methods developed with these data cannot differentiate biomass according to the tree species or tree age. ${ }^{7-9}$

We have identified two major problems in biomass estimation by remote sensing classification techniques. The first problem is the noise in the image classification on VHR data. Figure 1 shows "the salt-pepper noise problem" on the Para rubber plantation mapping. The Thaichote image [Fig. 1(a)] was classified with a classical pixel based technique [Fig. 1(b)]. The result shows inaccuracies in terms of the surface geometry of the age class boundary (or plantation limit). The legacy technique is derived from supervised and unsupervised methods for assigning a class label to an individual pixel based on distance or similarity measures in feature space. ${ }^{14}$ This approach was used as simple spectral information for class identification.

(a)

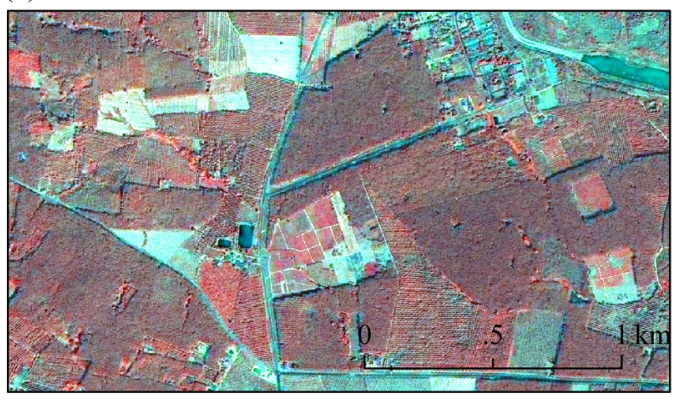

(b)

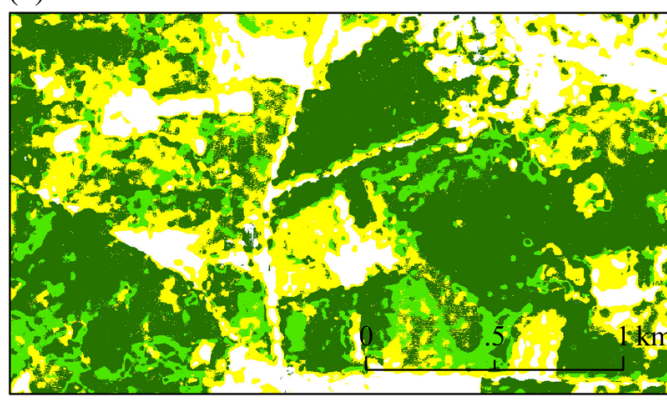

Para rubber age class map.

Young

Middle

Old

(c)

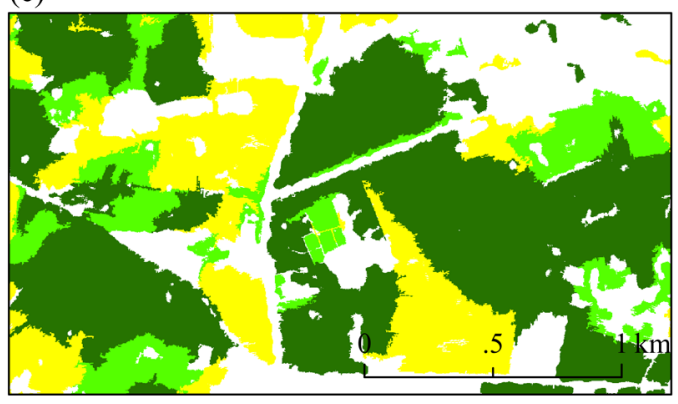

Fig. 1 (a) Thaichote false-color image (RGB: NIR, red, blue, spatial resolution of $2 \mathrm{~m}$ ). (b) Para rubber age-class map derived from pixel-based classification with coverage by "salt-pepper noise" and misclassification of some age classes of Para rubber plantations. (c) Para rubber age-class map derived from object-based classification. 
By contrast, the object-based classification [object-based image analysis (OBIA)] overcomes the limitation of pixel-based classification. Figure 1(c) revised the Para rubber plantation mapping derived from the OBIA approach. The OBIA process is done in two steps: image segmentation and modeling for object identification. Segmentation can remove the image noise while the model can identify the object by analyzing more information such as reflectance distribution, shape, size, and texture. ${ }^{14}$ The second problem is the poor-quality relationship between field data and VHR data when using spectral information for biomass estimation. Recently, methods based on the texture measurement were developed to obtain better results than those using only spectral information..$^{7-9}$ The texture of image is a good description of the forest canopy architecture. It was shown to have a certain relation with biomass volume. ${ }^{7-9}$ Classical pixel-based classification is not a good candidate to determine the characteristics of the canopy. Most of the previous works were used as simple spectral information for forest biomass estimation using medium resolution satellite images. ${ }^{11-13}$ Consequently, the established relationships between field data and remote sensing data were weak.

The goal of this study is to improve the Para rubber biomass and carbon stocks estimation using object-based classification combining both spectral and textural information from a Thaichote satellite image that was acquired in December 2011 over the Mae num Prasae watershed (Thailand). In the following, the study area and the Para rubber tree characteristics are first described. Then, the data and the remote sensing techniques are described and the results are given and commented on. Finally, the consequences of the results are discussed.

\section{Study Area and Para Rubber Plantation}

The area chosen in this study is the Mae num Prasae watershed located near $12^{\circ} 58^{\prime} 22^{\prime \prime} \mathrm{N} / 101^{\circ} 32^{\prime}$ 56 "E (Rayong province, East Thailand) covering a surface of $232 \mathrm{~km}^{2}$ (Fig. 2). The average elevation of the watershed is around $43 \mathrm{~m}$ above MSL and the average slope is 6 deg. Rainfalls occur around 120 days/year and the cumulative rainfall is $1900 \mathrm{~mm}$. The average temperature is $28^{\circ} \mathrm{C}$ and humidity ranges from $60 \%$ to $90 \%$. $^{15}$

The rubber clone of Hevea brasiliensis RRIM 600 (Rubber Research Institute of Malaysia No. 600) is planted in this area (Fig. 3). In 2011, 34\% of the Rayong province area was occupied

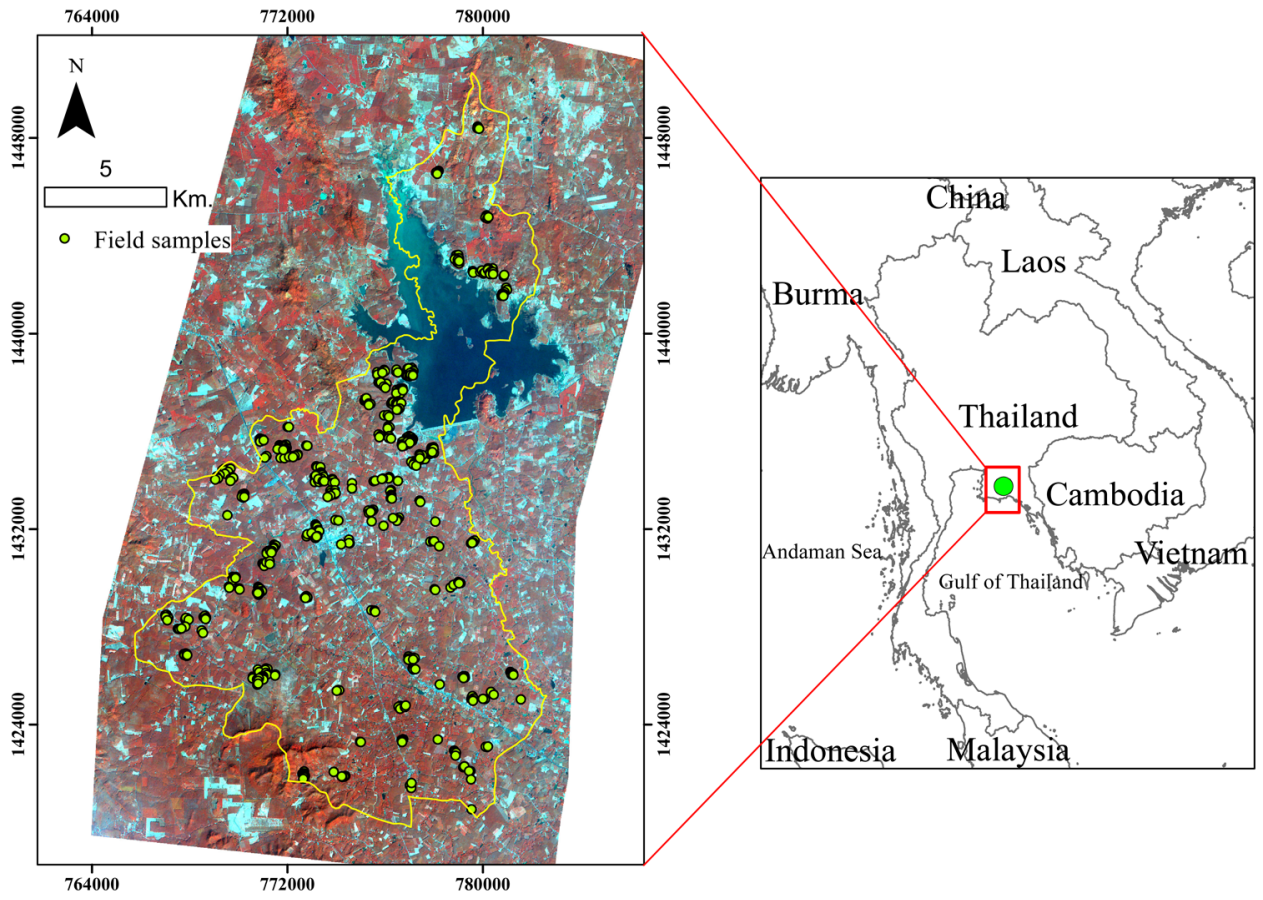

Fig. 2 The Mae num Prasae watershed in Thaichote satellite data (band composite RGB:NIR, red, green). The field data is shown in green dots. 

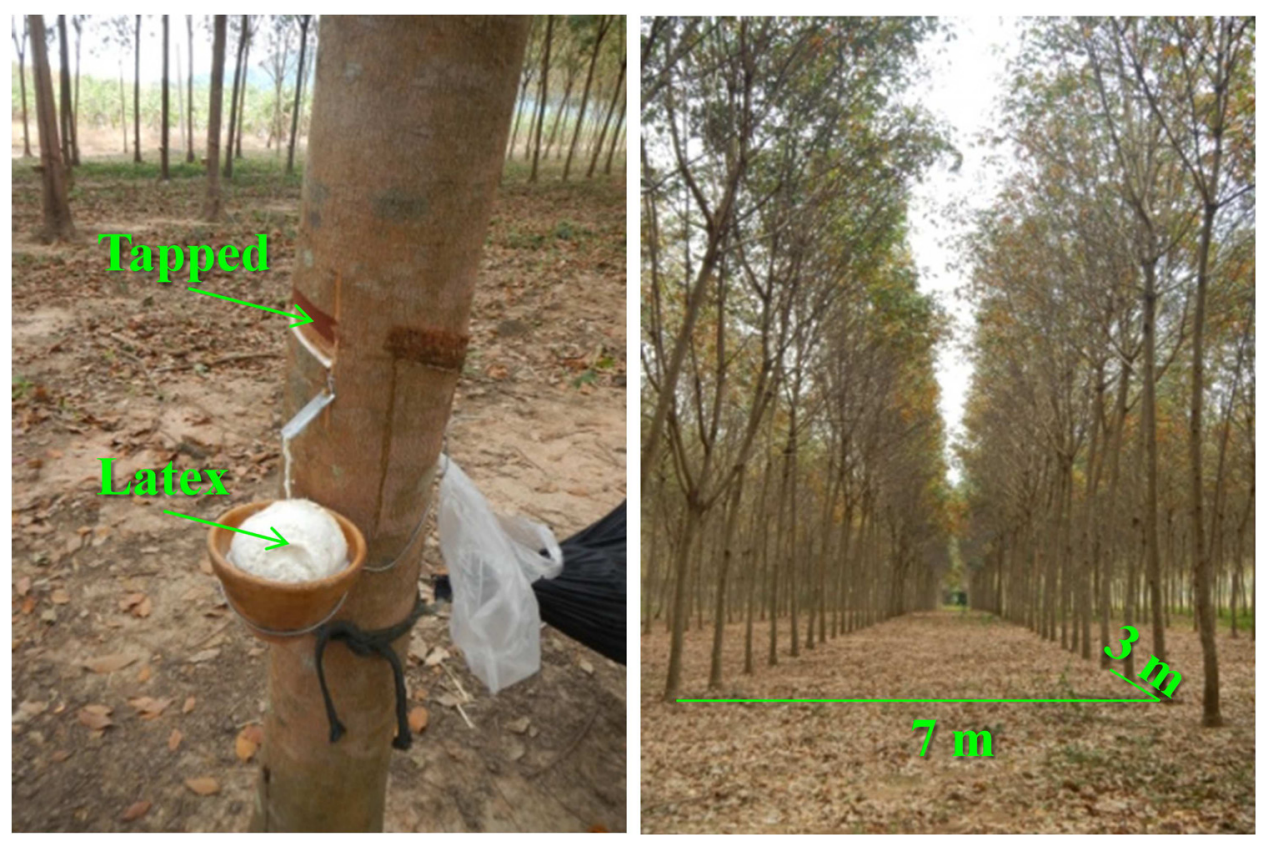

Fig. 3 Ground-view characteristics of Para rubber plantations, latex extraction and crop management (tree layout is approximately $3 \times 7 \mathrm{~m}$ ).

by Para rubber plantations. A rubber tree life cycle is around 25 to 30 years, after which the latex production from the rubber is decreased. Replanting is thus necessary to maintain latex production. Hevea brasiliensis needs a rainfall of $2000 \mathrm{~mm}$ (or more) with no severe dry season and 125 to 150 raining days/year. The minimum and maximum temperatures should be $20^{\circ} \mathrm{C}$ and $35^{\circ} \mathrm{C}$, respectively, and atmospheric humidity should be $80 \%$ to $90 \%$ with moderate wind and bright sunshine for about $2000 \mathrm{~h}$ a year. ${ }^{16}$

\section{Data and Methods}

Classical methods are based on identification of forest plantations from classification of spectral pixel information on remote sensing images. Our purpose is to improve the classification by OBIA by combining both spectral and textural information from the images. The method developed here needs field data that will permit the building of a transfer equation model between field and remote sensing data.

The girths of trees were measured in the field according to their age in 500 randomly chosen plots. In each plot, the characteristics of 10 to 15 trees were measured and evaluated. The biomass and the carbon storage for a single tree were estimated from already published allometric equations. ${ }^{17,18}$ In parallel, the Thaichote satellite image $(2 \mathrm{~m})$ was corrected from atmospheric artifacts and then classified according to the OBIA method. ${ }^{14}$ The first step of OBIA consists of the image segmentation in order to identify the Para rubber plantations and to estimate the number of trees available per plantation. In a second step, textural and vegetation indices were constructed from the images using classical descriptors. The new images combined with the initial bands were used as inputs in a classification process to extract a numerical relation between the girths and ages of trees and the indicator values of the sampled plots. The empirical model obtained from a simple linear multiple regression technique was used to estimate the age of every plantation. Finally, the biomass and carbon stocked by Para rubbers in the Mae num Prasae watershed were estimated from the map of the plantation, the age estimated for each plantation and the relationship between the age and carbon stock for a single tree.

\subsection{Field Data}

In December 2011, field data were randomly collected on 500 sample plots (shown in Fig. 2 by green dots). In the field, the diameter at breast height (DBH), girth, and age were measured on 


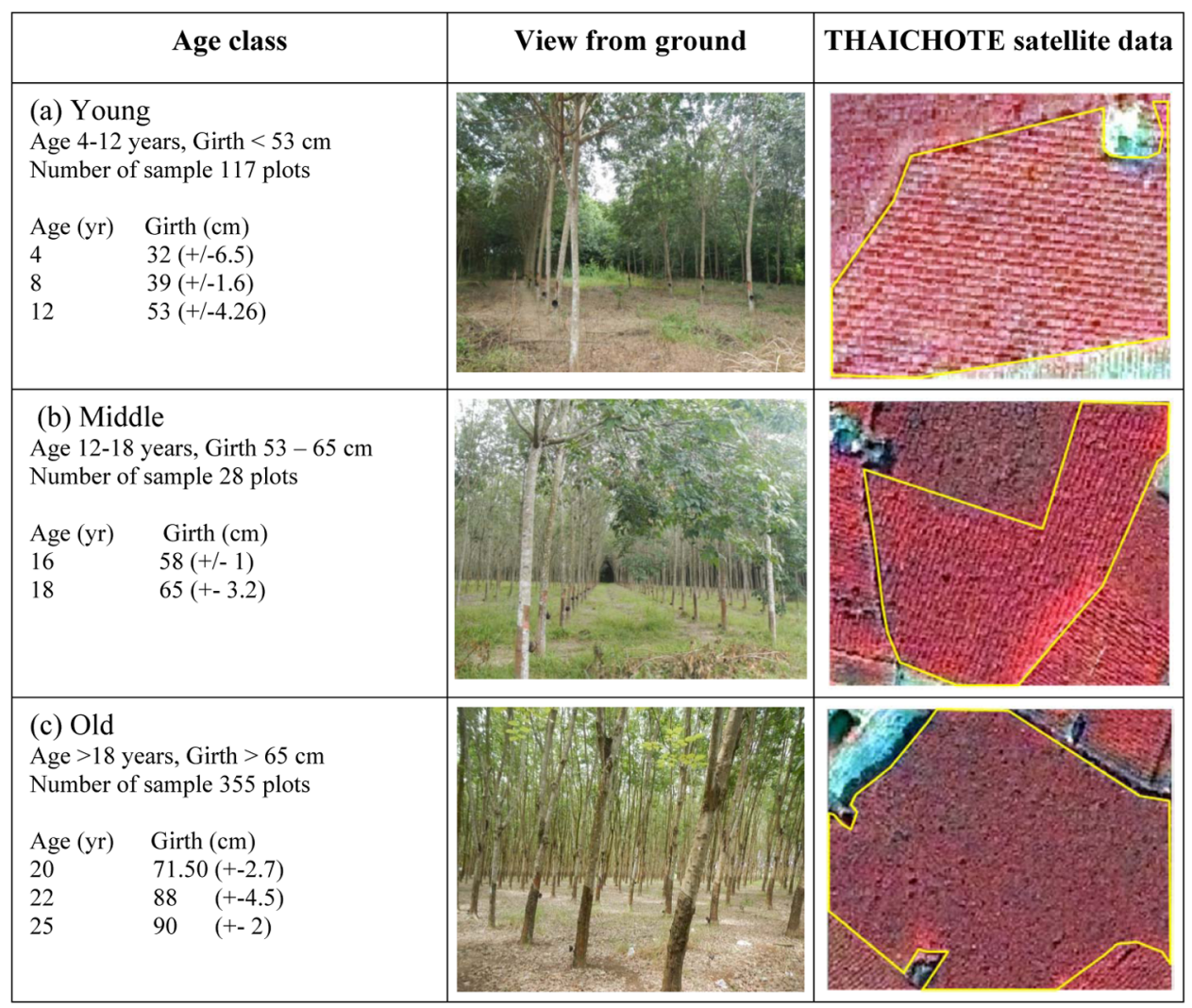

Fig. 4 Field data collected from December 2011 to April 2012: view from ground and view from Thaichote satellite data (band composite RGB: NIR, red, green) for the Para rubber ages used in this study.

about 10 to 15 trees per plot. Random plots were used for the forest inventory associated to tree age range from 4 to 25 years old. The age data were classified in eight classes (Fig. 4). DBH and tree girth were measured with a diameter tape at heights of 1.3 to $1.7 \mathrm{~m}$ above the ground according to the position of the latex tap on the skin of the trunk (Fig. 3). Cultivation of the Para rubber plantation has a traditional spatial distribution. Trees are spaced $3 \mathrm{~m}$ apart in lines spaced $7 \mathrm{~m}$ apart (Fig. 3). The density of a Para rubber tree stand is $\sim 76$ trees per $1600 \mathrm{~m}^{2}$ or 0.0475 tree $^{-2}$. Data were managed in a GIS database which included a topographic map and satellite image projected in the Universal Transverse Mercator (UTM) 48N Zone on World Geodetic System 1984 (WGS84). The Para rubber plantation statistics are shown in Fig. 4.

\subsection{Ground Biomass and Carbon Estimation}

Biomass estimation was derived from the empirically allometric equation relating geometric parameters of trees to their biomass and carbon content. The study used the allometric equation proposed by Chantuma et al. ${ }^{18}$ specifically for Para rubber to estimate the biomass Eq. (1):

$$
Y=0.0082 X^{2.5623},
$$

where $Y$ is the tree dry biomass in $\mathrm{kg}$ and $X$ is the girth of a tree in $\mathrm{cm}$. The coefficient of correlation $\left(R^{2}\right)$ is 0.96 . This equation was developed by measurements realized on plots located in the North, North East, South and East of Thailand. The carbon mass of a given tree is proportional to the biomass by a conversion factor of $0.4452 .{ }^{18}$ The rate of carbon sequestration (in $\mathrm{tCha}^{-1} \mathrm{y}^{-1}$ ) is given by the following Eq. (2): 
Table 1 Thaichote instrument characteristics. Thaichote was launched on October 2008.

\begin{tabular}{lcc}
\hline \hline Characteristics & \multicolumn{1}{c}{ Multispectral } & Panchromatic \\
\hline Spectral range & $\begin{array}{l}\text { Blue band } 0.45 \text { to } 0.52 \mu \mathrm{m} . \text { Green band } 0.53 \text { to } 0.60 \mu \mathrm{m} . \\
\text { Red band 0.62 to } 0.69 \mu \mathrm{m} . \text { NIR band } 0.77 \text { to } 0.90 \mu \mathrm{m} . \\
\text { (Nadir looking) }\end{array}$ & $\begin{array}{c}0.45 \text { to } 0.90 \mu \mathrm{m} . \\
\text { (Nadir looking) }\end{array}$ \\
Spatial resolution & $15 \mathrm{~m}$ & $2 \mathrm{~m}$ \\
Swath width & $90 \mathrm{~km}$ & $22 \mathrm{~km}$ \\
Pixel depth & $8 \mathrm{bits}$ & 8 bits \\
\hline \hline
\end{tabular}

$$
\text { Carbon sequestration }=\frac{\text { Carbon mass in } 1 \text { ha }}{\text { Age of trees }},
$$

where carbon sequestration is the amount of carbon sequestered by each age class per year expressed as tons of $\mathrm{C}$ per hectare per year $\left(\mathrm{tC} \mathrm{ha}^{-1} \mathrm{yr}^{-1}\right)$. Carbon density is a total amount of carbon stored by each age (years) expressed as tons of $\mathrm{C}$ per hectare $\left(\mathrm{tC} \mathrm{ha}^{-1}\right)$.

\subsection{Thaichote Satellite Images Preprocessing}

The Thaichote satellite image at level 1A containing both multispectral (Table 1) and panchromatic data was acquired on December 27, 2011, at 03:22 GMT, during the dry season where cloud cover was $<10 \%$. The sun azimuth was $143.21 \mathrm{deg}$ and the sun elevation was $44.42 \mathrm{deg}$. The image was first pan-sharpened $(2 \mathrm{~m})$ and georeferenced ${ }^{19}$ in the Universal Transverse Mercator projection Zone 48 North on World Geodetic System 1984 ellipsoid (UTM WGS1984 Z48N) and corrected from topographic distortion using ASTER Global Digital Elevation (GDEM Version 2). The cosine of the solar zenith corrections ${ }^{20}$ was used to correct the radiometry of the image.

\subsection{Image Classification}

The image classification technique was used to map Para rubber plantations and estimate the age of each plantation. The technique developed here uses a combination of spectral information (data from spectral bands and band ratios called in the following vegetation indices), textural information and mask information.

Five classical vegetation indices obtained by Refs. 21-25 were calculated from the four spectral bands (Table 2). Each index is a combination of the various bands' ratios. The resulting complete spectral dataset contained nine layers.

The texture of an image is related to the statistical characteristics of association of pixels at a given scale. The texture of an image is a good descriptor of the forest canopy ${ }^{7-9}$ The gray-level co-occurrence matrix (GLCM) texture measurement ${ }^{26,27}$ was applied to the Thaichote image. A $15 \times 15$ pixels sliding window ${ }^{7}$ was used to generate a co-occurrence matrix. The Haralick et al. ${ }^{26}$ equations were used for building texture descriptors. These equations refer to three groups of descriptors that are the contrast group (contrast, dissimilarity, and homogeneity), the orderliness group (angular second moment, entropy), and the descriptive statistics group (mean, variance, and correlation) (Table 2). Eight textural layers were computed from the original image.

We also used the Thai National Spatial Data Infrastructure GIS database 2011 obtained by the Geo-Informatics and Space Technology Development Agency (GISTDA, Thailand) to extract the Para rubber plantation areas and evaluate the areas that not considered in the study: (1) the urban areas, other agricultural, natural forest areas and roads were selected for building mask information, (2) the mask database was map referenced to the UTM WGS84, Z48, (3) the localization of bare soil and water bodies was evaluated by the inverse of normalized different vegetation index. These areas were masked on the Thaichote pan-sharpened image. 
Table 2 Layers used for image classification.

\begin{tabular}{|c|c|c|}
\hline Image mining & Formula & References \\
\hline aSingle bands & Blue, Green, Red, NIR & \\
\hline${ }^{\mathrm{a} A R V I}$ & $\frac{\mathrm{NIR}-2 \text { (RED)-BLUE }}{\mathrm{NIR+2(RED)-BLUE}}$ & Ref. 21 \\
\hline${ }^{\mathrm{a} G E M I}$ & $\frac{n(1-0.25 n)-\mathrm{RED}-0.125}{1-\mathrm{RED}}$ where $n=\frac{2\left[\left(\mathrm{NIR}^{2}-\mathrm{RED}^{2}\right)\right]+1.5(\mathrm{NIR})}{1-\mathrm{RED}}$ & Ref. 22 \\
\hline aIPVI & $\frac{\mathrm{NIR}}{\mathrm{NIR+RED}}$ & Ref. 23 \\
\hline aMSAVI2 & $\frac{2 \mathrm{NIR}+1-\sqrt{(2 \mathrm{NIR}+1)^{2}-8(\mathrm{NIR}-\mathrm{RED})}}{2}$ & Ref. 24 \\
\hline${ }^{\mathrm{a}} \mathrm{NDVI}$ & $\frac{\text { NIR-RED }}{\text { NIR+RED }}$ & Ref. 25 \\
\hline${ }^{\mathrm{b}} \mathrm{GLCM}$ contrast (CON) & $f_{\mathrm{CON}}=\sum_{l, J=0}^{N-1} \mathrm{P}_{I, J}|i-j|^{2}$ & Ref. 26 \\
\hline${ }^{\mathrm{b}} \mathrm{GLCM}$ dissimilarity (DIS) & $f_{\mathrm{DIS}}=\sum_{i, j=0}^{N-1} P_{i, j}|i-j|$ & \\
\hline${ }^{\mathrm{b}} \mathrm{GLCM}$ homogeneity (HOM) & $f_{\mathrm{HOM}}=\sum_{i, j=0}^{N-1} \frac{P_{i, j}}{1+(i-j)^{2}}$ & \\
\hline${ }^{\mathrm{b}} \mathrm{GLCM}$ angular second moment (ASM) & $f_{\mathrm{ASM}}=\sum_{i, j=1}^{N-1} P_{i, j}^{2}$ & \\
\hline${ }^{b}$ GLCM entropy (ENT) & $f_{\mathrm{ENT}}=\sum_{i, j=0}^{N-1} P_{i, j}\left(-\ln P_{i, j}\right)$ & \\
\hline${ }^{\mathrm{b}} \mathrm{GLCM}$ mean & $f_{\mu_{i}}=\mu_{i} \sum_{i, j=0}^{N-1} i\left(P_{i, j}\right), f_{\mu_{j}}=\mu_{j} \sum_{i, j=0}^{N-1} j\left(P_{i, j}\right)$ & \\
\hline${ }^{b}$ GLCM variance & $f_{\text {variance }}=\sum_{i, j=0}^{N-1} P_{i, j}(i-\mu)^{2}$ & \\
\hline${ }^{b}$ GLCM correlation & $f_{\text {correlation }}=\sum_{i, j=0}^{N-1}\left[\frac{\left(i-\mu_{i}\right)\left(j-\mu_{j}\right)}{\sqrt{\left(\sigma_{i}^{2}\right)+\left(\sigma_{j}^{2}\right)}}\right]$ & \\
\hline
\end{tabular}

${ }^{a}$ For spectral information: NIR is near infrared band, ARVI is atmospherically resistant vegetation index, GEMI is global environment monitoring index, IPVI is infrared percentage vegetation index, MSAVI2 is modified soil adjusted vegetation index 2 and NDVI is normalized difference vegetation index.

${ }^{b}$ For textural information, GLCM: where $P_{i, j}=$ the probability matrix, $i=$ reference pixel, $j=$ neighborhood pixel and $\mu_{i}, \mu_{j}, \sigma_{i}, \sigma_{j}=$ the mean and standard deviation of $P_{i, j}$, respectively.

\subsection{Para Rubber Age Class and Tree-Girth Classification}

OBIA was used to classify the Para rubber tree canopy properties. A complete description of the OBIA is beyond the scope of the paper and can be found in Ref. 14. Two results were extracted from image classification and modeling which are a map of the plantations and an estimation of the girths and ages of the trees of each plantation. The map of the plantation was realized using an image segmentation process. The girths and ages of trees of each plantation were estimated from a linear regression equation built from the relationships between field data and image characteristics measured at the emplacement where field data were acquired.

Para rubber plantation limits were automatically extracted from image multiscale segmentation $^{28,29}$ on Thaichote data. After the multiscale segmentation, the limits of the plantations were obtained. The results of multiscale segmentation were tested by empirical visualization. ${ }^{29}$ Therefore, the total number of trees was estimated by GIS area calculation from the classical density of plantations.

Values of the various vegetation and textural indices were extracted at the position of each plot measured in the field. From that dataset and using a multiple linear regression model, a set of linear equations relating the girth of the tree and the characteristics of the layers was extracted in the form of Eq. (3). The confidence in this equation has been analyzed using Pearson's correlation coefficient.

The tree girth model (TGM) was generated using a linear multiple regression stepwise $\operatorname{method}^{7,8}$ for predicting tree girth and tree age. The tree girth is a function of multiparameterization of spectral and textural information. This is shown in Eq. (3):

$$
Y=\mathrm{a}+\left[b_{1} X_{1}+b_{2} X_{2} \ldots+b_{n} X_{n}\right],
$$

where $Y$ is Tree girth $(\mathrm{m})$, a is a constant, $\left(b_{1} b_{n}\right)$ are coefficients of image parameters, and $\left(X_{1 \ldots n}\right)$ are spectral and textural parameters (from four single bands, five vegetation indices, 
and eight GLCM). The mean value of these parameters was assigned to each plantation. The root mean square error (RMSE) and mean absolute percentage error (MAPE) were used to evaluate the model accuracy. Equation (3) was used to estimate the girth of trees of each plantation delineated at the segmentation step. Knowing the surface of the plantation and the age of the plantation, the biomass of each plantation was calculated from the allometric equation. Carbon stock was estimated using the conversion factor and carbon sequestration was estimated using Eq. (2).

\section{Results and Discussion}

\subsection{Map of Para Rubber Plantation Limits}

Ten thousand and sixty-nine crops of Para rubber plantation were identified with a maximum surface of $0.23 \mathrm{~km}^{2}$ and a minimum surface of $1600 \mathrm{~m}^{2}$, while the mean surface was $15,329 \mathrm{~m}^{2}$. The uncertainty of Para rubber plantation surface was calculated from a comparison of multiscale segmentation and manual digitization. The total surface of plantations is $154.34 \mathrm{~km}^{2}(15,434 \mathrm{ha})$ with an uncertainty of $11 \%\left( \pm 17 \mathrm{~km}^{2}\right)$. The number of Para rubber tree stands was calculated using a constant value of 475 trees ha $^{-1}$ obtained by field measurement. Thus, the total number of tree stands is approximately 7,321,444 trees ( $\pm 802,832$ trees). Figure 5 shows a sample of the map of Para rubber plantations obtained from the image segmentation process.

\subsection{TGM and Para Rubber Tree Classification}

The correlation between tree girth and values of the different layers obtained from the remote sensing image was tested by Pearson's correlation. All the parameters of texture and vegetation indices were correlated to the tree girth (Table 3 ). The homogeneity has the highest correlation with tree girth (0.875), while lowest is the mean (-0.496). For vegetation index, MSAVI2 has the highest correlation with tree girth $(-0.663)$, whereas ARVI has the lowest $(-0.512)$. For the bands of the satellite image, NIR (near infrared) is the band that correlates better with tree girth (correlation coefficient of -0.679).

Different models were built from different layers (Table 4). A first model (TGM\#1) used only the spectral information. The coefficient of correlation of this model was poor $(0.53)$. Thus, the

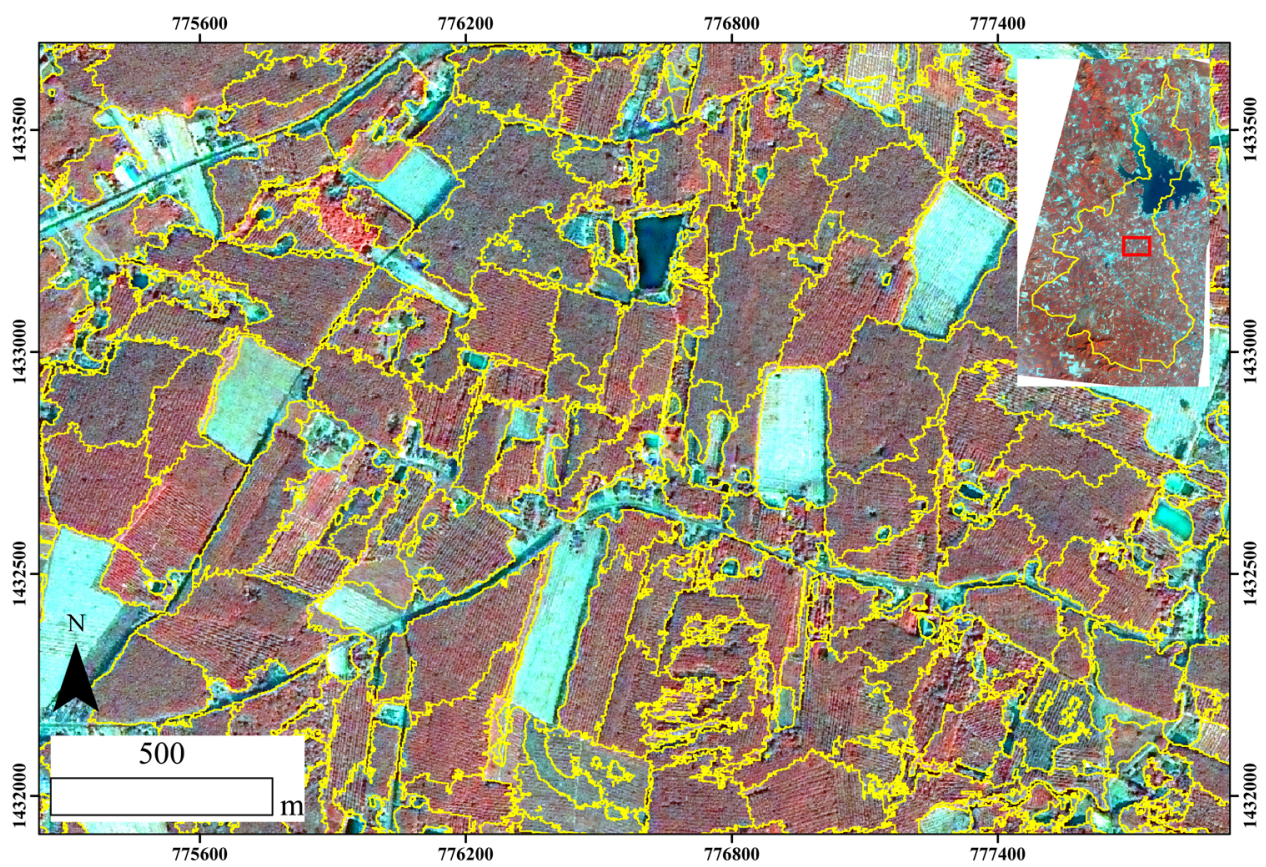

Fig. 5 Example of map of Para rubber plantations extracted from the multiscale segmentation. Yellow line is Para rubber plantation limits. 
model (TGM\#1) was rejected. The integration of the textural layers improved strongly improved the model. The better fit by TGM\#2 achieved a coefficient of regression of 0.87 . It used the layers GEMI, HOMO, DIS, CON and VAR. The equation of TGM\#2 is shown in Eq. (4):

$$
Y=3.694-1.29(\text { GEMI })-2.740(\mathrm{HOMO})-0.933(\mathrm{DIS})+0.068(\mathrm{CON})-0.015(\mathrm{VAR}),
$$

where $Y$ is the tree girth (m) and $R^{2}$ is 0.87 . The scatter plots of TGM\#1 and TGM\#2 are shown in Fig. 6.

This equation was applied to the layers defining each plantation in order to estimate the girth of the trees of each plantation. The model (TGM\#2) was realized in the GIS built for this study. From the tree girth map, a map of tree ages has been drawn (Fig. 7).

Old age classes (more than 18 years old) cover a surface of $54.55 \mathrm{~km}^{2}(35 \%, 5455 \mathrm{ha})$, Young age classes (less than 12 years old) cover a surface of $51.12 \mathrm{~km}^{2}(33 \%, 5112 \mathrm{ha})$, and middle age classes (from 12 to 18 years old) cover a surface of $48.68 \mathrm{~km}^{2}(32 \%$, 4868 ha). The areas of each age class are listed in Table 5.

The obtained relationships between tree girth and layers used for image classification show that texture parameters are better correlated than single bands and vegetation indices as shown by the Pearson's correlation coefficient values. The results of our study are in agreement with Eckert ${ }^{7}$ findings who observed the degraded forest stratum with high resolution WorldView-2 data.

The textures are better parameters than spectral data to estimate the age of the canopy. The present work shows that the textural information has to be added to the spectral one for a precise inventory of characteristics of forest or plantations in agreement with the works of Eckert ${ }^{7}$ and Sarker and $\mathrm{Nichol}^{8}$ with high resolution WorldView-2 and ALOS AVNIR-2 used in Madagascar, Hong Kong and Central Siberia for forest biomass modeling.

Table 3 Pearson's correlation between tree girth and layer parameters measured at field plot positions. Only the layers with an absolute value of correlation coefficient better than 0.4 were integrated in the final multilinear regression model.

\begin{tabular}{lcc}
\hline \hline Parameter & Pearson's correlation coefficient & Sig. \\
\hline GLCM homogeneity & 0.875 & 0.000 \\
GLCM entropy & -0.853 & 0.000 \\
GLCM dissimilarity & -0.841 & 0.000 \\
GLCM angular second moment & 0.803 & 0.000 \\
GLCM variance & -0.801 & 0.000 \\
GLCM contrast & -0.787 & 0.000 \\
GLCM correlation & 0.754 & 0.000 \\
NIR & -0.679 & 0.000 \\
MSAVI2 & -0.663 & 0.000 \\
GEMI & -0.633 & 0.000 \\
IPVI & -0.540 & 0.000 \\
NDVI & -0.540 & 0.000 \\
ARVI & -0.512 & 0.000 \\
GLCM mean & -0.496 & 0.000 \\
Red & 0.215 & 0.000 \\
Blue & 0.083 & 0.052 \\
Green & 0.014 & 0.389 \\
\hline \hline
\end{tabular}


Table 4 Model summary. The number of girth measurements is 388 samples. Method: Stepwise regression, Criteria $=$ Probability in (0.05), Probability out(.10). Spectral information; NIR= near-infra red band, RED = red band, GEMI = global environment monitoring index, MSAVI2 = modified soil adjusted vegetation index 2 . Textural information; HOMO = homogeneous, DIS = dissimilarity, $\mathrm{CON}=$ contrast, $\mathrm{VAR}=$ variance.

\begin{tabular}{lccccccc}
\hline \hline Model & $R^{2}$ & Adj. $R^{2}$ & $\begin{array}{c}\text { RMSE tree } \\
\text { girth }(\mathrm{cm})\end{array}$ & $\begin{array}{c}\text { RMSE } \\
\text { biomass } \\
(\mathrm{t} / \mathrm{ha})\end{array}$ & $\begin{array}{c}\text { RMSE } \\
\text { carbon } \\
(\mathrm{tC} / \mathrm{ha})\end{array}$ & $\begin{array}{c}\text { MAPE } \\
(\%)\end{array}$ & \multicolumn{1}{c}{ Coefficient } \\
\hline TGM\#1 & 0.531 & 0.526 & 11.26 & 1.93 & 0.86 & 15.43 & $\begin{array}{l}\text { (Constant) }-16.435 \mathrm{NIR}-95.441 \\
\text { RED 73.826 MSAVI2 55.867 } \\
\text { GEMl 18.064 }\end{array}$ \\
TGM\#2 & 0.865 & 0.863 & 6.05 & 0.39 & 0.17 & 8.33 & $\begin{array}{l}\text { (Constant) 3.694 GEMI }-1.290 \\
\text { HOMO -2.740 DIS }-0.933 \\
\text { CON 0.068 VAR }-0.015\end{array}$ \\
\hline \hline
\end{tabular}
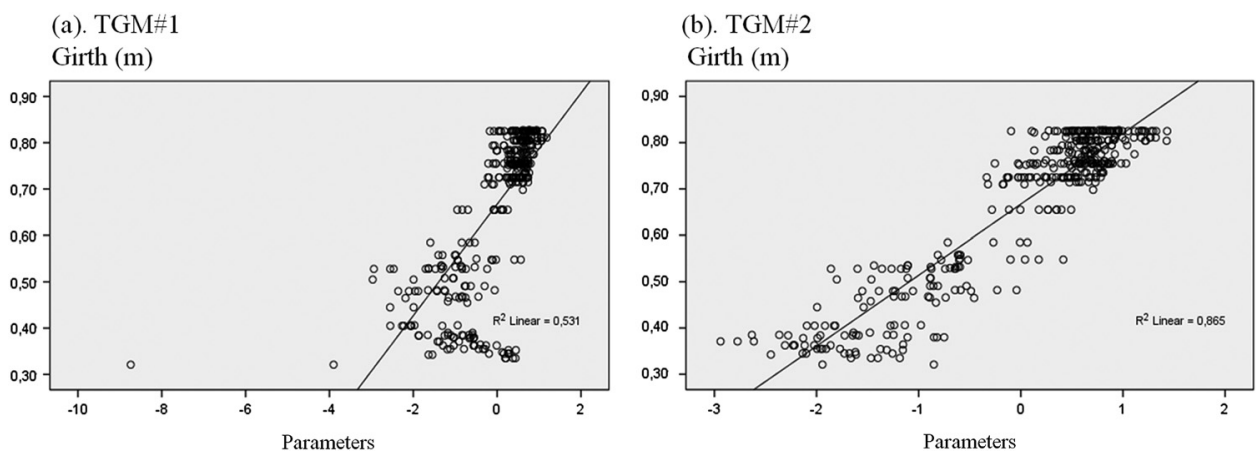

Fig. 6 (a) TGM\#1 derived from spectral information. (b) TGM\#2 derived from a combination of spectral and textural information.

Table 5 Para rubber classification, biomass and carbon stock in study area 2011. The uncertainty on the carbon stock comes from the uncertainty on the surface and the uncertainty of the model.

\begin{tabular}{lccccccc}
\hline \hline Class & $\begin{array}{c}\text { Age } \\
(\mathrm{yr})\end{array}$ & $\begin{array}{c}\text { Area } \\
(\mathrm{ha})\end{array}$ & $\begin{array}{c}\text { Biomass } \\
\text { stock }(\mathrm{t})\end{array}$ & $\begin{array}{c}\text { C Stock } \\
(\mathrm{tC})\end{array}$ & $\begin{array}{c}\text { Uncertainty } \\
\text { of C stock } \\
(\mathrm{tC})\end{array}$ & $\begin{array}{c}\text { C sequestered } \\
\left(\mathrm{tC} \mathrm{ha}^{-1} \mathrm{yr}^{-1}\right)\end{array}$ & $\begin{array}{c}\mathrm{CO}_{2} \text { sequestered } \\
\left(\mathrm{tCO}_{2} \mathrm{ha}^{-1} \mathrm{yr}^{-1}\right)\end{array}$ \\
\hline Young & 4 & 52.05 & $1,275.55$ & 567.88 & 72.16 & 2.73 & 10.01 \\
& 8 & 899.26 & $34,309.54$ & $15,274.61$ & $1,845.45$ & 2.12 & 7.79 \\
& 12 & $4,160.34$ & $311,479.13$ & $138,670.51$ & $15,987.87$ & 2.78 & 10.19 \\
Middle & 16 & $1,965.42$ & $223,062.92$ & $99,307.61$ & $11,363.43$ & 3.16 & 11.59 \\
& 18 & $2,902.70$ & $436,121.53$ & $194,161.30$ & $21,820.70$ & 3.72 & 13.64 \\
Old & 20 & $2,658.79$ & $514,323.60$ & $228,976.87$ & $25,588.01$ & 4.31 & 15.8 \\
& 22 & $2,791.13$ & $703,766.39$ & $313,316.80$ & $34,847.56$ & 5.1 & 18.73 \\
& 25 & 5.17 & $2,654.43$ & $1,181.75$ & 131.13 & 9.14 & 33.53 \\
\multicolumn{2}{l}{ Grand total } & $15,434.87$ & $2,226,993.09$ & $991,457.32$ & $111,656.32(11.3 \%)$ & 33.05 & 121.28 \\
\hline \hline
\end{tabular}

\subsection{Estimation of Biomass and Carbon Stocks}

The total amount of biomass stock in the study area was estimated using the field data and remote sensing data. The field data and Eq. (4) were used to predict the girth and age of every plantation. Then the biomass stock was calculated using the allometric Eq. (1). In the study area, the highest biomass stock in Para rubber plantations is obtained for 22 years old trees that sequester 
Charoenjit et al.: Estimation of biomass and carbon stock in Para rubber plantations...

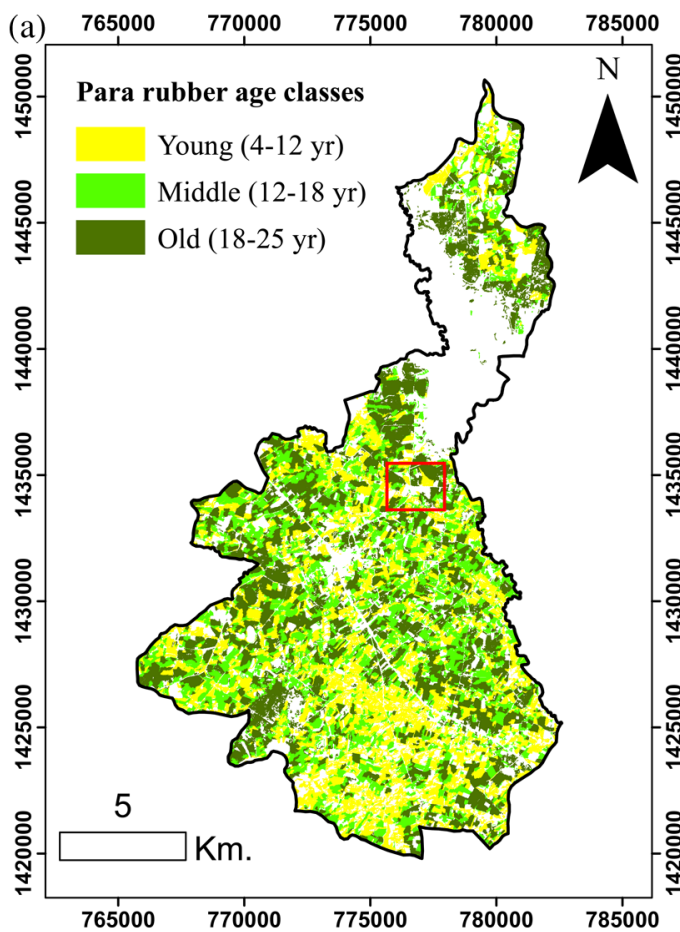

(b)

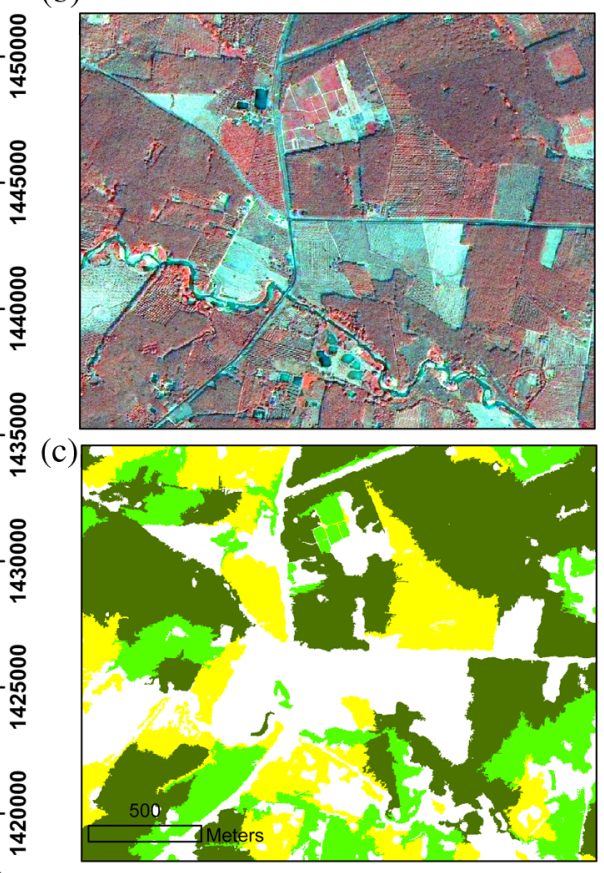

Fig. 7 (a) Map of Para rubber age classes. (b) Thaichote false color-image. (c) Zoom map of Para rubber age classes.

approximately $32 \%$ of the total biomass (703, 766 tons). The lower biomass stock is found at 4 years (approximate $0.1 \%$ or 1276 tons) while at the age classes of $20,18,12,16,8$ and 25 years, the amount of biomass stocked is $23 \%, 20 \%, 14 \%, 10 \%, 1 \%$ and $0.1 \%$ respectively (Table 5, Fig. 8). The carbon stock map is given in Fig. 9. The total biomass stock in the study area is 2.23 Megatons corresponding to 0.99 Megatons of carbon stock.

The accuracy of the model was evaluated using the RMSE and the MAPE. The RMSE and MAPE are $0.17 \mathrm{tC} \mathrm{ha}^{-1}$ and $8.33 \%$, respectively. The errors from the surface and the model were summarized. Consequently, the total uncertainty of the carbon stock estimation is $111,656.32$ tons $(11.3 \%$ ) (Table 5).

The evaluation of $\mathrm{CO}_{2}$ sequestration by Para rubber trees by age is reported in Table 5 . We found that Para rubber has the higher $\mathrm{C}$ sequestration at 25 years $\left(33.53 \mathrm{tC} \mathrm{ha}^{-1} \mathrm{yr}^{-1}\right)$, whereas the lower $\mathrm{C}$ sequestration is found at 8 years $\left(7.79 \mathrm{tC} \mathrm{ha}^{-1} \mathrm{yr}^{-1}\right)$. In 2011 , the investigated area sequestered $33.05 \mathrm{tC}$ corresponding to $121.28 \mathrm{t} \mathrm{CO}_{2}$ by Para rubber plantation assuming that $1 \mathrm{tC}$ represents $3.676 \mathrm{t}$ of $\mathrm{CO}_{2}$.

(a)

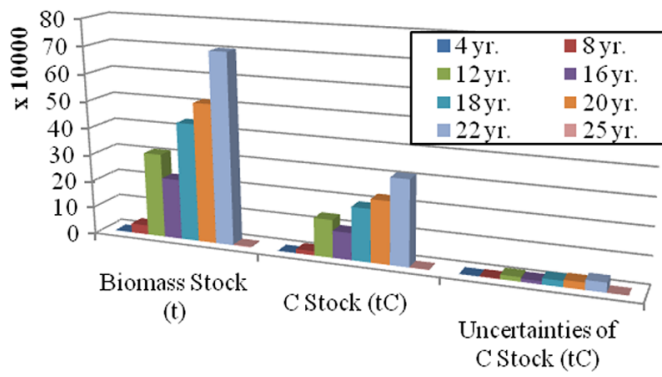

(b)

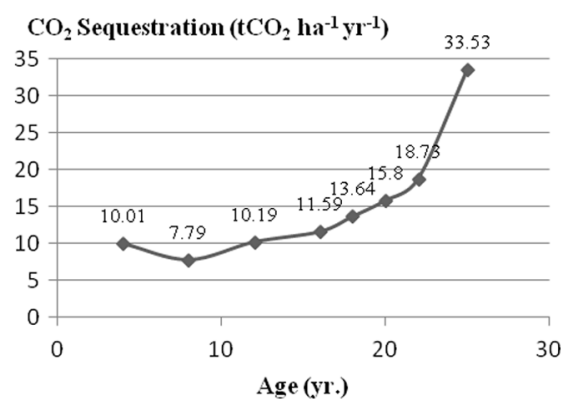

Fig. 8 (a) Bar graphs: biomass, carbon stock, and uncertainties data. (b) The rate of $\mathrm{CO}_{2}$ sequestration by Para rubber of each age class. 


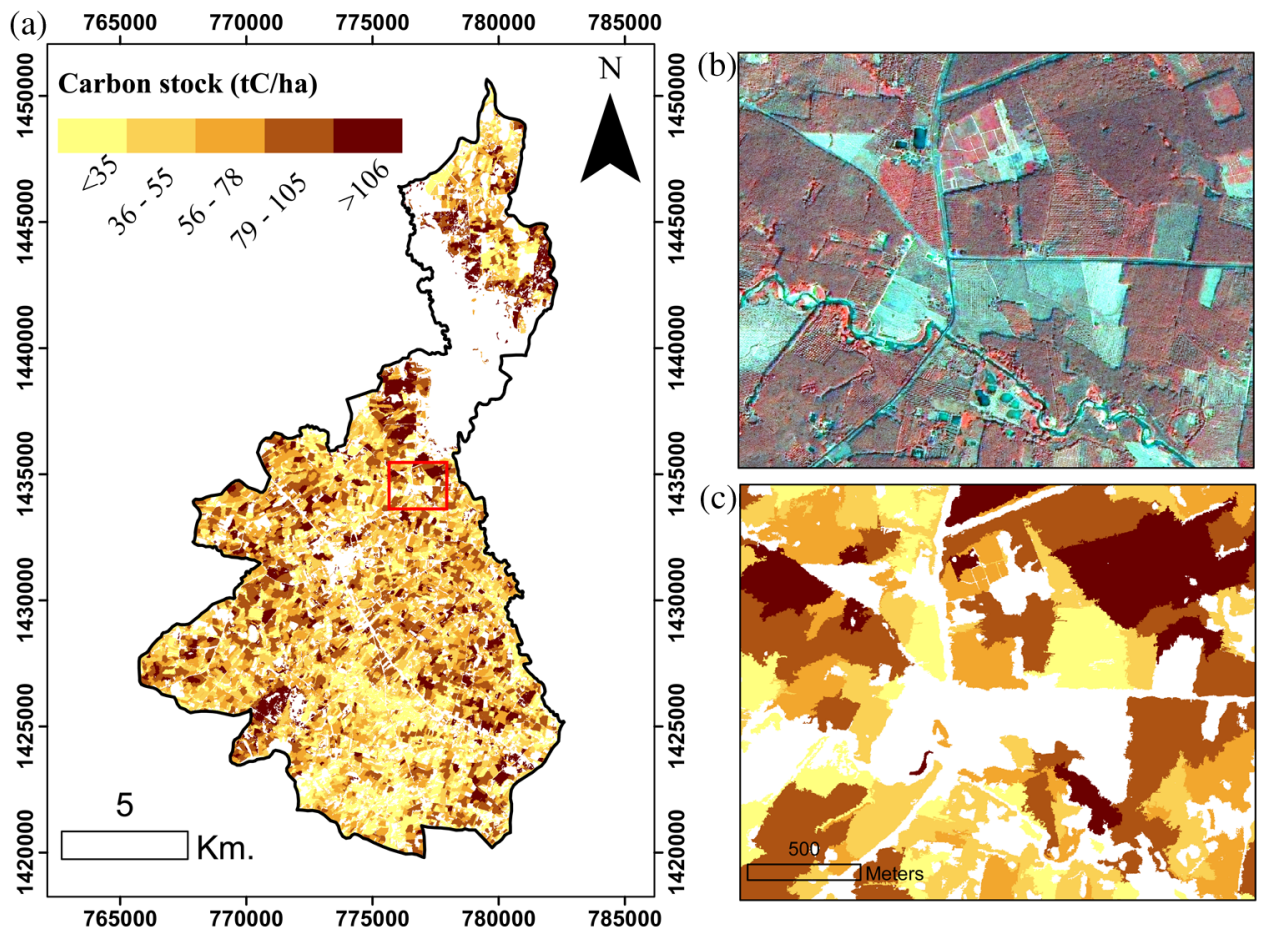

Fig. 9 (a) Map of Para rubber carbon stock. (b) Thaichote false color-image. (c) Zoom map of Para rubber carbon stock.

The result of our estimation confirms the strong potential of Para rubber for $\mathrm{CO}_{2}$ capture as suggested by Chuntuma et al. ${ }^{18}$ based on the tree physiological characteristics. Our results show that the rate capture of $\mathrm{CO}_{2}$ is $33.53 \mathrm{tCO}_{2} \mathrm{ha}^{-1} \mathrm{yr}^{-1}$ that is on the same order of magnitude of the values of $\mathrm{CO}_{2}$ capture found in Ghana (35.30 tCO $\left.\mathrm{ha}^{-1} \mathrm{yr}^{-1}\right)$, Malaysia $\left(38.33 \mathrm{tCO}_{2} \mathrm{ha}^{-1} \mathrm{yr}^{-1}\right.$ ) and Indonesia (29.30 $\left.\mathrm{tCO}_{2} \mathrm{ha}^{-1} \mathrm{yr}^{-1}\right)$ for Para rubber. ${ }^{30}$

These amounts of $\mathrm{CO}_{2}$ captured by Para rubber plantations can be compared to natural carbon sequestration estimated for the ocean. Borges et al. ${ }^{31}$ show that the sequestration of $\mathrm{CO}_{2}$ by the ocean is around $4.45 \mathrm{gC} \mathrm{m}^{-2} \mathrm{yr}^{-1}$, while Para rubber plantation can sequester up to $914.3 \mathrm{gC} \mathrm{m}^{-2} \mathrm{yr}^{-1}$. We believe that agriculture and human intervention may play a critical role in the extraction of $\mathrm{CO}_{2}$ from the atmosphere and thus in the short carbon cycle.

\section{Conclusions and Further Research}

This study explored the potential of Thaichote satellite data to estimate Para rubber biomass and carbon stock. Despite the fact that Thaichote data do not contain medium infrared data (MIR) as used in other studies, ${ }^{32,33}$ the results of our study have shown a high potential for forest biomass evaluation. The Para rubber plantation is a non-evergreen forest type. In the study area, the leaves of Para rubber fall between February and May. The method developed in this paper considered green plantations. Additional work remains to be done to test the potential of Thaichote data acquired during the period when trees have no leaves.

The results of this study show that these data can be used to map Para rubber plantations and distinguish the age classes of trees in the plantations. We propose that textural information is more useful than spectral information to capture tree canopy architecture and thus the age of the canopy. Moreover, it has been possible to build a model equation relating some textural parameters to the age of the plantation. This equation has been obtained from multiple linear regression analysis with a correlation coefficient of 0.87 and thus can be used with confidence on the study area. Around $154 \mathrm{~km}^{2}$ of the $232 \mathrm{~km}^{2}$ of the studied area are covered by Para rubber plantations. The class of age for each plantation has been estimated as follows: $33 \%$ of the crop surface belongs to the young class (from 4 to 12 years), $34 \%$ of the crop surface belongs to the middle class (from 12 to 18 years), and 33\% belongs to the old class (older than 18 years). The total amount of biomass and carbon stock is 2.23 Megatons and 0.99 Megatons C, respectively, with 
an uncertainty of $11 \%$. In 2011, the total area sequestered $121 \mathrm{tCO}_{2}$ by Para rubber plantations. Such a value is two orders of magnitude higher than the carbon sequestered in the ocean.

\section{Acknowledgments}

We would like to thank the Geo-Informatics and Space Technology Development Agency (GISTDA) for providing Thaichote satellite data without charge, and the team of GIREN, Mahidol University and Faculty of Forestry, Kasetsart University for the help on establishing the biomass and carbon forest inventory. We also thank two anonymous reviewers and the associate editor Dr. Feng Gao for suggestions that improved the quality and the clarity of the manuscript.

Financial support was partially provided through a scholarship to Kitsanai Charoenjit by the Franco-Thai program from Pierre et Marie Curie and Burapha Universities.

\section{References}

1. R. Sales, D. Lasco, and M. R. Banaticla, "Carbon storage and sequestration potential of smallholder tree farms on Leyte Island, the Philippines," in ACIAR Smallholder Forestry Project ASEM 200/008 Redevelopment of a Timber Industry Following Extensive Land Clearing: Proceedings from the End-of Project Workshop, Ormoc City, Philippines (2004).

2. S. Backeus, P. Wikstrom, and T. Lamas, "A model for regional analysis of carbon sequestration and timber production," For. Ecol. Manage. 216, 28-40 (2005).

3. R. Sedjo, "Forest carbon sequestration: some issues for forest investment," Discussion Paper 01-34, Resources for the Future (2001).

4. "United Nation Framework Convention on Climate Change, Kyoto Protocol," http://unfccc .int/2860.php (20 October 2014).

5. C. Viriyabuncha, Handbook of Stand Biomass Estimation, pp. 1-11, Department of National Parks, Wildlife and Plant Conservation, Thailand (2003).

6. "Production of Natural Rubber," Rubber Research Institute of Thailand, 2011, http://www .rubberthai.com/statistic/stat_index.htm accessed (20 October 2014).

7. S. Eckert, "Improved forest biomass and carbon estimations using texture measures from WorldView-2 Satellite data," Remote Sens. 4, 810-829 (2012).

8. L. R. Sarker and J. E. Nichol, "Improved forest biomass estimates using ALOS AVNIR-2 texture indices," Remote Sens. Environ. 115, 968-977 (2011).

9. H. Fuchs et al., "Estimating aboveground carbon in a catchment of the Siberian forest tundra: combining satellite imagery and field inventory," Remote Sens. Environ. 113, 518-531 (2009).

10. D. Lu et al., "Relationships between forest stand parameters and Landsat TM spectral responses in the Brazilian Amazon basin," For. Ecol. Manage. 198, 149-167 (2004).

11. C. O. Iglesias, "Determination of carbon sequestration and storage capacity of eucalyptus plantation in Sra Kaew Province," Thailand using remote sensing, MSc Thesis, Mahidol University, Thailand (2007).

12. J. Nilubol, "Geo-informatics technology for age class identification for Para rubber plantation in Krabi province," MSc Thesis, Mahidol University (2007).

13. G. Zheng et al., "Combining remote sensing imagery and forest age inventory for biomass mapping," J. Environ. Manage. 85, 616-623 (2007).

14. L. Arko, "Uncertainties in segmentation and their visualisation application of geoinformatics," PhD Thesis, Universiteit Utrecht (2004).

15. "Annual weather summary of Thailand in 2011," Thai Meteorological Department http:// www.tmd.go.th/programs\%5Cuploads\%5CyearlySummary\%5CAnnual2011_up.pdf accessed (October 20 2014).

16. P. Rao and K. R. Vijayakumar, "Climatic requirements," in Natural Rubber: Biology, Cultivation and Technology, Developments in Crop Science, Vol. 23, Elsevier, Netherlands (1992).

17. N. Picard, L. Saint-André, and M. Henry, Manual for Building Tree Volume and Biomass Allometric Equations, Food and Agriculture Organization of the United Nations (2012).

18. A. Chantuma, T. Wichitchonlachai, and P. Chantuma, "Rubber new planting in thailand: towards the world affected on climate change," Rubber Thai. 1, 40-47 (2012). 
19. G. Jacek and D. Gene, "Block adjustment of high-resolution satellite images described by rational polynomials," Photogramm. Eng. Remote Sens. 69(1), 59-68 (2003).

20. P. S. Chavez, Jr., "Image-based atmospheric corrections-revisited and revised," Photogramm. Eng. Remote Sens. 62(9), 1025-1036 (1996).

21. Y. J. Kaufman and D. Tanré, "Atmospherically resistant vegetation index (ARVI) for EOSMODIS," IEEE Trans. Geosci. Remote Sens. 30, 261-270 (1992).

22. B. Pinty and M. M. Verstraete, "GEMI: a non-linear index to monitor global vegetation from satellites," Vegetation 101, 15-20 (1992).

23. R. E. Crippen, "Calculating the vegetation index faster," Remote Sens. Environ. 34, 71-73 (1990).

24. J. Qi et al., "Modified soil adjusted vegetation index (MSAVI)," Remote Sens. Environ. 48, 119-126 (1994).

25. J. W. Rouse et al., "Monitoring vegetation systems in the great plains with ERTS," in Third ERTS Symposium, NASA SP-351, Vol. 1, pp. 309-317, NASA, Washington, DC (1973).

26. R. M. Haralick, K. Shanmugan, and I. Dinstein, "Textural features for image classification," IEEE Trans. Syst. Man Cybern. 3, 610-621 (1973).

27. H. Anys et al., "Texture analysis for the mapping of urban areas using airborne MEIS-II images," in Proc. First Int. Airborne Remote Sens. Conf. Exhibition, pp. 231-245, Strasbourg, France (1994).

28. L. Guigues, J.-P. Cocquerez, and H. Le Men, "Scale sets image analysis," Int. J. Comput. Vision 68(3), 289-317 (2006).

29. A. Le Bris, "Extraction of vineyards out of aerial ortho-image using texture information," in ISPRS Annals of the Photogrammetry, Remote Sensing and Spatial Information Sciences, XXII ISPRS Congress, Volume I-3, Melbourne, Australia (2012).

30. IRRDB, "The effects of climate change on NR cultivation and productivity," International Rubber Research and Development, Malaysian Rubber Board Building, Malaysia (2009).

31. A. V. Borges, B. Delille, and M. Frankignoulle, "Budgeting sinks and sources of $\mathrm{CO}^{2}$ in the coastal ocean: diversity of ecosystems counts," Geophys. Res. Lett. 32, L14601 (2005).

32. D. Lu, "Aboveground biomass estimation using Landsat TM data in the Brazilian Amazon," Int. J. Remote Sens. 26, 2509-2525 (2005).

33. S. A. Eckert, "Contribution to sustainable forest management in Patagonia: object-oriented classification and forest parameter extraction based on ASTER and Landsat ETM+ data," PhD Thesis, University of Zurich, Zurich, Switzerland (2005).

Kitsanai Charoenjit is a PhD candidate in the Institute of Sciences de la Terre de Paris at UPMC-Sorbonne Universités. His research focuses on forest carbon sequestration with emphasis on remote sensing technology and unmanned aerial systems (UAS). He is also an associate researcher at the Faculty of Geoinformatics, Burapha University.

Pierpaolo Zuddas is a professor of geochemistry in the Institute des Sciences de la Terre de Paris at UPMC-Sorbonne Universités. He made substantial contributions on carbon sequestration processes in both natural and artificial conditions. Prior to joining Sorbonne Universités, he was chair of the Institute of Environmental Engineering and Eco-Development and director of the Environment and Risk Program at the University of Lyon.

Pascal Allemand is a professor of earth sciences and remote sensing in the Laboratoire de Géologie de Lyon: Terre, Planète, Environnement at Universite de Lyon 1. His main research concerns the development of methods for landscape monitoring using remote sensing data, geospatial modeling, LiDAR, and unmanned aerial systems (UAS).

Sura Pattanakiat is an associate professor of forestry ecology and remote sensing and director of the Geo-Informatics in Resource and Environment Research and Training Center (GIREN), Mahidol University. His main research concerns development methods for land use and land cover on watershed monitoring and natural-disaster management using geoinformation.

Katavut Pachana is an assistant professor of the Department of Chemistry at Burapha University. His research is focused on environment and risk management. 